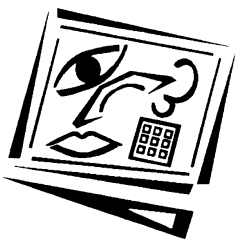

\title{
Educators' perceptions of automated feedback systems
}

\author{
Justin C. W. Debuse, Meredith Lawley and Rania Shibl
}

University of the Sunshine Coast

\begin{abstract}
Assessment of student learning is a core function of educators. Ideally students should be provided with timely, constructive feedback to facilitate learning. However, provision of high quality feedback becomes more complex as class sizes increase, modes of study expand and academic workloads increase. ICT solutions are being developed to facilitate quality feedback, whilst not impacting adversely upon staff workloads. Hence the research question of this study is 'How do academic staff perceive the usefulness of an automated feedback system in terms of impact on workloads and quality of feedback?' This study used an automated feedback generator (AFG) across multiple tutors and assessment items within an MBA course delivered in a variety of modes. All academics marking in the course completed a survey based on an adaptation of the unified theory of acceptance and use of technology (UTAUT) model. Results indicated that while the workload impact was generally positive with savings in both cost and time, improvements and modifications to the system could further reduce workloads. Furthermore, results indicated that AFG improves quality in terms of timeliness, greater consistency between markers and an increase in the amount of feedback provided.
\end{abstract}

\section{Introduction}

The educational impact of ICT has risen dramatically in recent years, from lecture recording and transcription systems through online delivery systems and plagiarism detectors. Although many innovations have provided benefits to students and educational organisations, such as improving flexibility and geographical reach, their effect on educators can be less positive. Whilst ICT can reduce teaching workloads (Selwood \& Pilkington, 2005), the overheads associated with learning and using some technologies may result in a net workload increase for those responsible for teaching courses (Selwood, 2004, cited in Selwood \& Pilkington, 2005) or require compensatory reductions in teaching load (Bongalos, Bulaon, Celedonio, Guzman \& Ogarte, 2006). However, this imbalance may be addressed by technologies that offer net efficiency gains to educators in time consuming tasks.

One of the most demanding tasks faced by educators is the production of feedback for students. Students should be given a good quantity of feedback (Holmes \& Smith, 2003) that is informative (James, McInnis \& Devlin, 2002), specific (Higgins, Hartley \& Skelton, 2002), personalised (Higgins et al., 2002), timely (James et al., 2002; Wiggins, 1997), consistent (Holmes \& Smith, 2003), detailed (Wiggins, 1997) and legible (Higgins et al., 2002). Feedback may be in written or oral forms (Debuse, Lawley \& Shibl, 2007; McCormack \& Taylor, 2006), containing elements such as comments, marks and performance relative to peers (Bower, 2005; Debuse et al., 2007). Feedback can also be generated for individuals and groups, and by educators, peers and students 
themselves (Parikh, McReelis \& Hodges, 2001). Assessment feedback is known to be important in higher education (Higgins et al., 2002), and is valued by students (Weaver, 2006), particularly in individual, group and peer form (Parikh et al., 2001). Although further research is required regarding the use of feedback by students (Higgins et al., 2002; McCormack \& Taylor, 2006), it has the potential to be applied to their future work in direct or reflective modes (Higgins et al., 2002).

Feedback can be produced using either manual or automated approaches (Debuse et al., 2007). Fully automated systems can perform the entire grading and feedback generation process automatically, but this restricts their application to specific assessment tasks such as spreadsheets (Blayney \& Freeman, 2004) or essays (Williams \& Dreher, 2004). Systems such as Re:Mark (Re:Mark, 2006), the Electronic Feedback System (EFS) (Denton, 2001) and MindTrail (Cargill, 2001), that automate only the feedback production, achieve far greater flexibility and thus appear to offer the best compromise between efficiency and the range of assessment types to which they may be applied. Such systems have the potential to allow educators, often provided with very limited resources, to provide quality feedback.

Existing studies of automated feedback from an educator's perspective have been qualitative and limited in scope; examples exist where the number of educators examined is two (Stevens \& Jamieson, 2002) and eight (Cargill, 2001), although in the latter only their students were investigated. These suggest for the MindTrail system that, although the learning curve is steep and setting up can exceed four hours, this is counterbalanced by marking consistency and feedback quality improvements (Cargill, 2001; Stevens \& Jamieson, 2002). Hence the purpose of this study is to further explore staff perceptions of usefulness of an automated feedback system specifically in terms of workloads and quality of feedback.

This study begins by developing the propositions to be tested and introducing the Automated Feedback Generator (AFG) system, followed by the research method used and results produced. Results are then discussed, followed by the conclusions and proposals for future research.

\section{Propositions}

Whilst several alternative models exist to explain the decision making process involved in adopting new products, in the context of an ICT application this research focussed upon technology adoption frameworks. One useful framework to explore the potential impacts of an automated feedback system on both staff workloads and quality of feedback is the unified theory of acceptance and use of technology (UTAUT) (Venkatesh, Morris, Davis \& Davis, 2003). The UTAUT model is based on the eight prominent technology acceptance models: Diffusion of Innovations, Technology Acceptance Model, Theory of Reasoned Action; Theory of Planned Behaviour, Combined TRA \& TPB, Motivational Model, PC utilisation model and the Social Cognitive Theory. The UTAUT collapses these eight key IT acceptance models into four main constructs: performance expectancy, effort expectancy, social influence and facilitating conditions, each of which has a significant positive impact on user acceptance and usage behaviour (Venkatesh et al., 2003). The UTAUT can explain up to $70 \%$ of the variance in intention, compared to $30-40 \%$ for alternative models (Meister \& Compeau, 2002; Venkatesh \& Davis, 2000; Venkatesh et al., 2003). The UTAUT constructs encompass the key issues of workloads and quality as outlined below. 
Performance expectancy is job performance enhancement by the user that is attributable to their use of the technology (Venkatesh et al., 2003). In terms of automated feedback, performance expectancy relates to the quality of feedback provided, as well as improvements in the time and cost of work. Hence our first proposition is that staff will perceive that an automated feedback system allows the provision of higher quality feedback while simulataneously saving both time and costs.

Effort expectancy relates to the usability of the technology (Venkatesh et al., 2003). Within an automated feedback system, this would refer to the ease of its installation as well as use once installed, again impacting on workloads. Ideally, to be accepted by users and have the desired result of positively impacting on workloads, an automated system should be easy to both install and use. The level of effort required to use and install an ICT system such as AFG will be influenced by the level of expertise and experience an educator has in relation to ICT, with more experienced staff, such as those teaching in Information Systems, expected to expend far less effort in installing and using the system than those staff with less expertise and experience. Hence our second propositon is that staff should perceive an automated feedback system to be easy to install and use, however we further propose that this would be influenced by the amount of expertise in ICT of the staff involved.

Social influence is the perception by the user that important people believe that they should use the technology (Venkatesh et al., 2003). This construct has a direct influence on intention to use the technology when its use is mandatory. However, in voluntary situations this merely impacts on the user's perception of the technology (Venkatesh \& Davis, 2000). The social influence construct exists in only some of the technology acceptance models, and was added to further explain the use of technology in voluntary versus involuntary use (Venkatesh et al., 2003); thus, the likelihood of social influence being of little importance to technology use in voluntary situations does not render the UTAUT model any less efficient in predicting intention. Within the academic culture, marking with an automated feedback system would generally not be mandatory, however markers in this study were initially given no choice and were required to use the system. Given this mandatory use of AFG, our third proposition was that staff would believe they should use the technology. However, as usage was mandatory we further proposed that social influence would be of little importance in their overall evaluation of the system.

Facilitating conditions relate to the support mechanisms that the user believes exist for use of the technology (Venkatesh et al., 2003). For an automated feedback system, these are any conditions that aid in its use. They are primarily instructions and technical support, as well as any prior knowledge that the user may have about using the system. Again these conditions would impact on the total workload involved in using an automated feedback system. This leads to our final proposition that staff will perceive that they have sufficient support to use an automated feedback system.

The automated feedback system examined using the UTAUT model is AFG since, unlike the now defunct MindTrail (Cargill, 2001), AFG has been found to produce comparable results to manual approaches from a student perspective (Debuse et al., 2007); whereas MindTrail proved comparable to manual feedback in terms of the improvements in marks gained by students from using the feedback on subsequent assessments, but was not examined from the perspective of student perceptions (Cargill, 2001). Moreover, AFG has been designed to minimise the overheads incurred 
through learning and using it, as well as the time and effort required to customise it for a specific assignment.

\section{The AFG system}

The AFG system was originally created by one of the authors using Microsoft Word's Visual Basic for Applications (VBA) (Debuse et al., 2007); however, preliminary usage across multiple markers suggested that differences in Word installations limited the extent to which the system could be used. A revised, platform independent version was therefore created in Java; this is illustrated in Figure 1. The system stores student details and marks in a table, with rows corresponding to students and columns to student details and marking criteria. Each criterion column must be initialised with a name, maximum number of marks and description; the student IDs and names can then be entered either manually or via import from the University's Blackboard system.

\begin{tabular}{|c|c|c|c|c|c|c|c|c|c|}
\hline \multicolumn{7}{|l|}{ AFG } & $\square[$ & & \\
\hline \multicolumn{10}{|c|}{ File View Help } \\
\hline \multicolumn{10}{|c|}{ Assessment name } \\
\hline \multicolumn{10}{|c|}{ Draft of Background and Problem Definition of Research Proposal } \\
\hline \multicolumn{6}{|c|}{ Student details and marks } & Criterion information & & & \\
\hline \multirow{3}{*}{\begin{tabular}{|l|} 
Student ID \\
123456 \\
654321 \\
\end{tabular}} & Student Na... & Student Sur.. & Background & Manageme.. & \multirow{3}{*}{$\mid \begin{array}{l}\mathrm{R} \\
\frac{2}{3} \\
3\end{array}$} & \multicolumn{4}{|l|}{ Background } \\
\hline & John & Smith & 3 & 1 & & \multicolumn{4}{|l|}{ Maximum Marks: 5} \\
\hline & Julie & Jones & 2 & 4 & & \multicolumn{4}{|l|}{ Description } \\
\hline & & & & & & \multicolumn{4}{|l|}{ Must encompass all information relevant to the proposal } \\
\hline 1 & III & 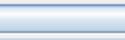 & 1 & & D & 4 & & - & 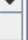 \\
\hline
\end{tabular}

Figure 1: The AFG system

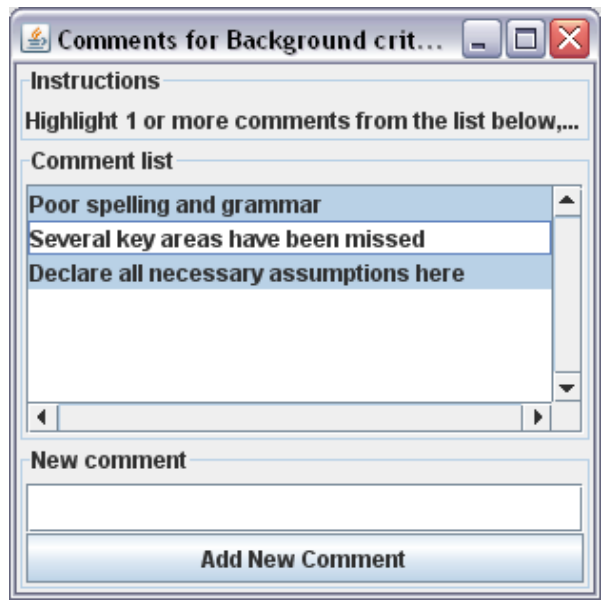

Figure 2: The AFG system comments form

Once the mark table has been initialised, marks can be entered into the criteria columns for each student, and comments may be added using the form shown in Figure 2. The form allows users to select from a list of all the comments entered for the criterion across all students, sorted so that the most popular appear at the top, and 
individual markers may also enter and add new comments. Unlike the earlier VBA version, the comments can be exported and imported, allowing feedback to be reused or standardised comments to be provided.

Marking criteria columns can optionally be formed into groups, using the form shown in Figure 3. Each group is given a title and optional comments; the group is used to link related criteria together into sections. For example, an assessment may contain criteria of currency and relevancy, both of which are to be placed together into a references section; a new group named references would therefore be created using the form in Figure 3, and the currency and relevancy criteria would be added to it. Students would then receive their currency and relevancy marks in a separate section of the feedback document entitled 'references'.

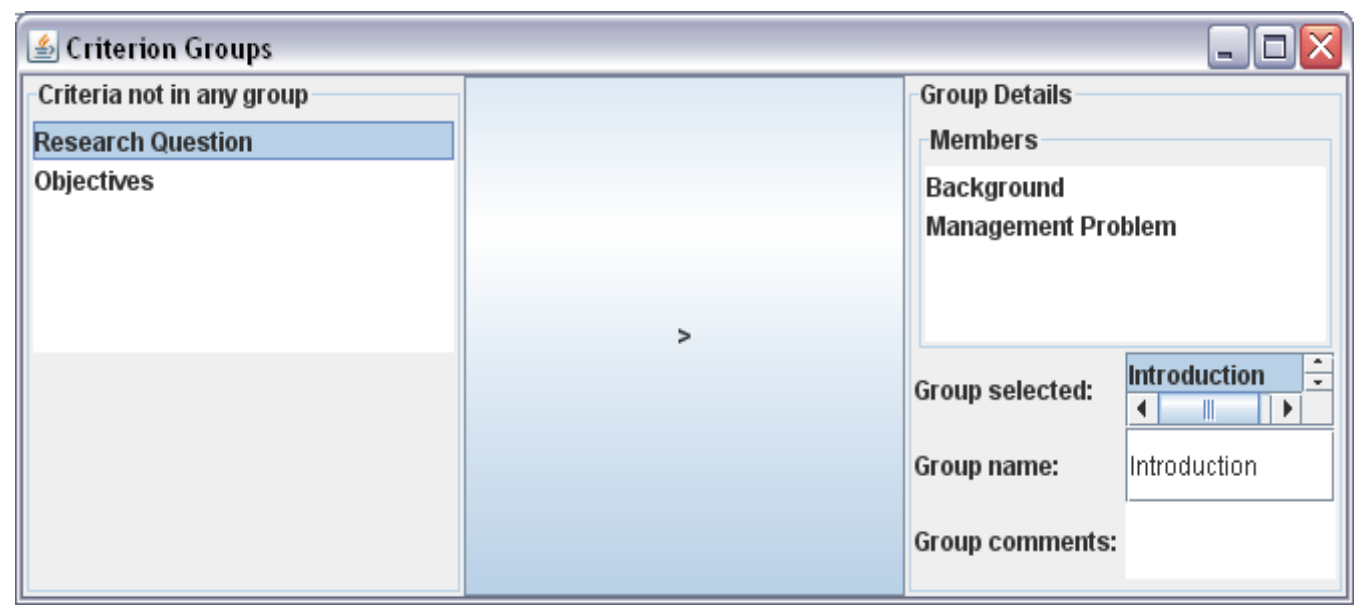

Figure 3: The AFG system mark column groups form

When marking is complete, the mark sheet can be exported as a spreadsheet or in a Blackboard compatible file for direct upload. A feedback document can also be generated for each student, containing their marks and, if required, comments in RTF, PDF, HTML or text format. In response to student and preliminary staff feedback, the marks are presented as positive numbers awarded rather than the deductions used in the VBA version. An example feedback document is given in Appendix 1.

\section{Method}

This study examines the use of the AFG system within a postgraduate MBA course containing approximately 300 students and delivered using the Blackboard system to online and on campus students. All eight educators involved in marking were required to use AFG, supported with installation and training on or off campus as appropriate, and AFG was used to mark three separate essay based assignments. The AFG system was prepared for the markers by one of the authors. For each assessment item, a mark sheet was created and a copy of this file containing the students to be marked was emailed to each marker. Two of the mark sheets contained preset comments entered by the course coordinator. When marks and feedback had been completed using AFG and moderated by the course coordinator they were uploaded to Blackboard. 
On completion of the course the markers were surveyed to determine the performance of the AFG system from a staff viewpoint and its potential for wider adoption. The survey, presented in full within Appendix 2, contained four major sections. The first section of the instrument identified characteristics of the educator likely to affect their attitudes and requirements from AFG, such as their competence with information and communications technology (ICT), experience with online marking and employment category. The second section determined the extent to which AFG was used within their marking, together with the amount of time required to learn and use the system.

The third section of the survey addressed the four constructs of the UTAUT using five point Likert scales measuring agreement with a series of statements, with potential values ranging from one (strongly disagree) through three (neutral) to five (strongly agree), together with zero (not applicable). Each statement belonged to one of the four constructs: Effort expectancy, Performance expectancy, Social influence and Facilitating conditions.

Three questions addressed the Performance expectancy construct, and all related to the relative advantage perceived as a result of using AFG. The specific advantages were measured in terms of time (C12), cost (C13) and work output (C14). Four questions addressed the Effort expectancy construct. These questions aimed to identify whether the AFG system was easy to use; they related to ease of use in general $(C 3, C 4)$ as well as the simplicity of installation (C5), and whether the use of AFG took time from other work as a result of requiring more effort (C1). Question C6 represented the Social influence construct, and explored the influence of colleagues on AFG usage. Six questions addressed the Facilitating conditions construct for the usage of AFG; these covered issues such as whether the user had the knowledge to use AFG (C7), the instructions on using AFG were helpful (C9, C10, C11) and the use of AFG fitted with the user's organisation and work style (C2, C8). The final two questions (C15 and C16) related to the users' intention to use AFG in the future or recommend its usage to others. These items do not relate to a specific construct, but responses were likely to be representative of the overall response to the other 14 questions within this section.

The final section of the instrument contained free response items allowing the best aspects of AFG to be identified, together with those aspects most in need of improvement; these comments were subsequently examined using thematic analysis. Finally, a five point Likert scale was used to rate AFG overall, with values ranging from one (very poor) through three (satisfactory) to five (very good).

\section{Results}

All eight educators involved in the course supplied responses, representing a response rate of $100 \%$. All but two of the respondents were very experienced, with at least seven years of university level teaching and marking. However, the maximum online marking experience was only six years. All but three educators worked on campus; two were full time and six sessional. The average level of ICT competence was moderate, although levels ranged from low to very high. Responses to the questions regarding specific aspects of the use and acceptance of AFG are summarised in Table 1.

Our first proposition, related to performance expectancy, was that staff will perceive that the AFG system allows the provision of higher quality feedback while simultaneously saving both time and costs. Generally the results support this proposition in relation to all three areas, with staff finding the greatest savings in 
relation to costs (mean 4.83), followed by time (mean 4.5) with improvements in quality positively perceived by all respondents except one who was unable to use the system (mean 3.5). Indeed, if the respondent who was unable to use the system is excluded, this proposition is supported even more strongly, with cost, time and quality means of $4.83,4.71$ and 3.86 respectively.

Table 1: Use and acceptance of AFG

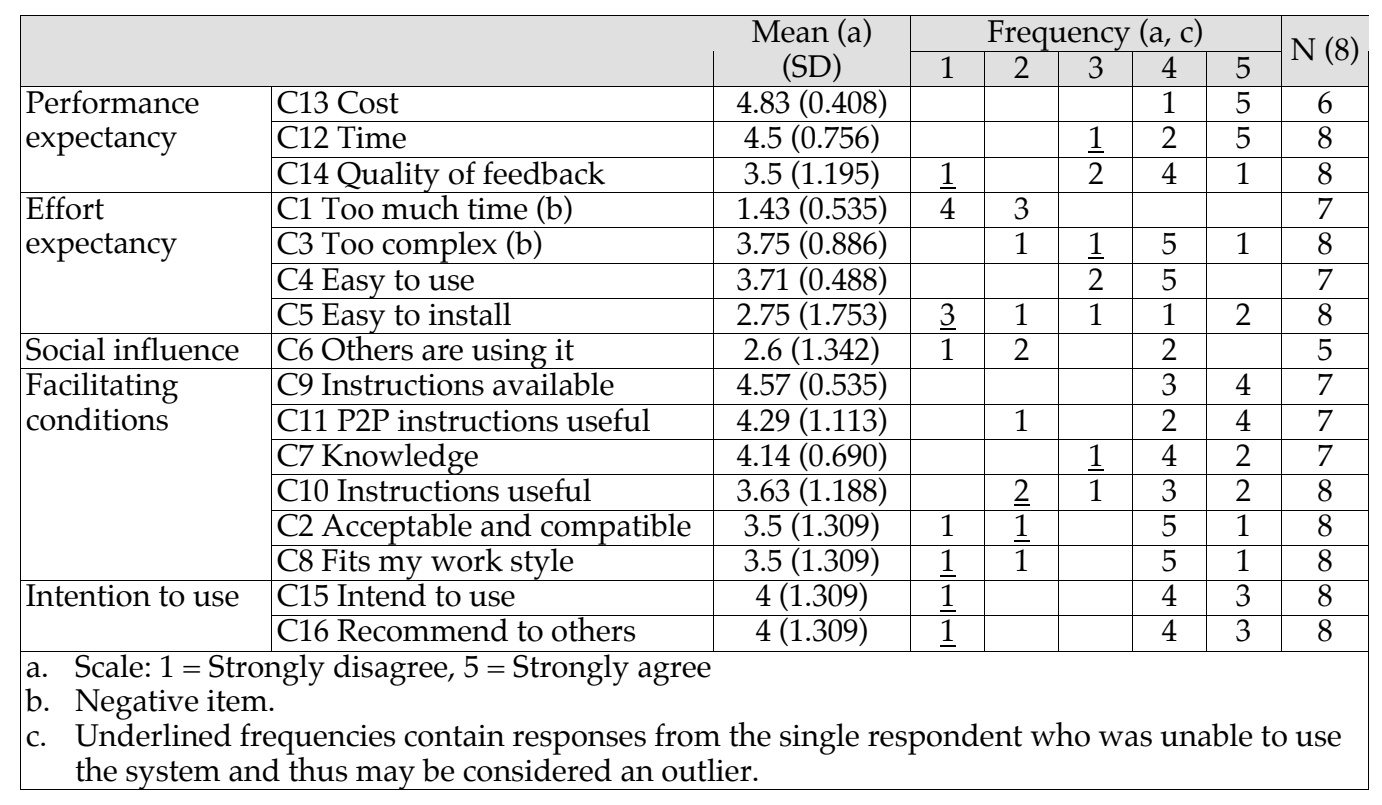

Our second propositon, related to effort expectancy, was that staff should perceive AFG to be easy to install and use; however, we further proposed that this would be influenced by the ICT expertise of the staff involved. Attitudinal data addressing this proposition is summarised in Table 1. In addition, respondents were asked for specific times spent installing and using AFG.

The installation of AFG proved to its most challenging aspect, with three of the eight respondents appearing unable to perform this without assistance and the remainder reporting times of up to four to five hours. One of the off campus educators was unable to complete installation of the system and did not seek additional assistance; their responses to items that required use of AFG were therefore excluded. The difficulty of installing AFG is further supported by the attitudinal responses where on average respondents disagreed (mean 2.75) that it was easy to install; indeed, this issue generated the strongest disagreement across all perceptions tested. Unsurprisingly, installation difficulty increased as ICT competence decreased. Respondents who found AFG difficult to install had only low or moderate ICT competence, whilst the remainder had moderate to very high competence.

The seven educators who had AFG installed used it to mark all of their allocated students, which ranged from 30 to 90 students per marker. Learning to use AFG proved significantly easier than installation; three respondents required 15 minutes or less, whilst the remainder took one to two hours. Higher levels of ICT competence appeared to reduce the learning duration. The attitudinal responses gave moderate 
support for the ease of use, and again were strengthened by higher ICT competence. These responses also suggest that AFG is too complex to learn and use without assistance (mean 3.75), although higher ICT competence reduces this effect.

The time requirements for AFG were its strongest effort expectancy aspect, with respondents tending to agree that usage did not take too much time from their normal duties, with the level of agreement increasing with ICT competence. Entering marks proved to be very rapid, taking less than one minute per student in two cases and at most thirty minutes for the remainder. Generating feedback was also fast, with no more than ten minutes per student being required, and higher levels of ICT competence had some effect on reducing this time.

In summary in relation to effort expectancy, the results suggest that while AFG is difficult to install, and this process can take several hours, once installation has been completed it is not time consuming to use. The person to person assistance given for initial installation and use was valuable, as the system was too complex to learn and use without it. However, it appears to be reasonably easy to use once learned. Higher levels of ICT competence appear to improve ease of use and installation of the system.

Our third proposition (social influence) was that staff would believe they should use the technology. However, as using AFG was mandatory we further proposed that social influence would be of little importance in their overall evaulation of the system. While seven out of eight educators used the system, in general they disagreed (mean 2.6) that their use was influenced by others. Of the four UTAUT constructs overall, social influence appeared to have the least impact on usage, with only one construct of effort expectancy (complexity) receiving more negative results.

Finally, in relation to facilitating conditions, we proposed that staff would perceive that they have sufficient support to use AFG. The results generally support this proposition, with specialised instruction (mean 4.57), person to person instruction (mean 4.29) and users' possession of knowledge necessary to use AFG (mean 4.14) being particularly strong. The written instructions (mean 3.63), compatibility with existing conditions (mean 3.5) and the users' work styles (mean 3.5) proved to be more marginal.

Supporting these quantitative responses, the qualitative responses to D1 suggest that time saving is the best aspect of AFG, allowing students to receive feedback more rapidly. The ability to reuse comments, together with the improvements in feedback consistency and quality, also proved valuable. The usefulness of the facility to 'seed' the system with comments was also commented on, along with the system usability; reducing paper consumption and suitability for printed and online assignments were also viewed as system strengths. The performance and effort expectancy constructs were therefore found to be qualitatively most positive.

Most of the responses to D2, the drawbacks of the system, concerned usability issues, such as the requirement to enter all marks before feedback is produced, screen crowding and requests for the addition of features such as a totals column, enhanced comment viewing and row and column tracking; improved instructions were also requested. A number of improvements to the commenting facility were suggested: comment ordering by selection order rather than alphabetical; spell checking (including a UK dictionary); comment editing, deletion and locking; and a facility to 
add an overall comment. One respondent preferred to use personalised feedback rather than individual comments, and another found it difficult to link comments to specific sections within the student's assignment. Further responses to D2 covered software bugs or misuse related problems. The effort expectancy and facilitating conditions constructs were therefore found to be qualitatively most negative.

The overall rating of AFG was very positive, with all but one educator responding 'good' or 'very good'; only the off campus educator who could not install the system rated it as 'poor'. The respondents also appeared keen to use AFG again in the future (mean 4) and recommend the system to others (mean 4).

In summary, the responses suggest that AFG is not time consuming to use, although the system is too complex to learn and use without person to person assistance. However, it appears to be reasonably easy to use once learned. Although AFG can be extremely difficult and time consuming to install, once this has been achieved the subsequent cost and time benefits of AFG appear compelling and it has moderate advantages over other options in terms of feedback quality. In relation to the four constructs of UTAUT, performance expectancy and facilitating conditions were positively perceived overall whereas effort expectancy and social influence had less positive outcomes.

The results can also be analysed using an approach similar to Debuse, Lawley \& Shibl (2007) and Stevens \& Jamieson (2002), where agreement and disagreement are denoted by responses of more than three and three or less respectively. The responses for agreement and disagreement are calculated using the lower and upper bound respectively of the $95 \%$ confidence interval for the mean response. This approach shows agreement through $95 \%$ confidence interval lower bound values for statements C3 (3.01), C4 (3.26), C7 (3.5), C9 (4.08), C11 (3.26), C12 (3.87), C13 (4.4); disagreement through upper bound values only occurred for $\mathrm{C} 1$ (1.92). These results therefore suggest that there is agreement with the following statements: two out of the three performance expectancy (cost and time); two out of the four effort expectancy (time and ease of use); and three out of the six facilitating conditions (knowledge, specialised instruction and person-to-person instruction). However, the $\mathrm{C} 3$ question (complexity) is negative, so agreement with it effectively counterbalances one of the effort expectancy items. The performance expectancy and facilitating conditions UTAUT constructs were thus effectively supported, whilst effort expectancy was to a limited extent and social influence was not. The lower bound of the 95\% confidence interval for the overall rating of AFG is 3.23, corresponding to a result between 'satisfactory' and 'good'.

\section{Discussion}

The key objective for this research was to investigate the perceptions of educators toward an automated feedback generator specifically in terms of staff workload impact and student feedback quality. The results indicated that while the workload impact was generally positive with savings in both cost and time, improvements and modifications to the system could further reduce workloads. Specifically, improvements related to initial installation and training would significantly reduce the time required to be able to start using the system. These results suggest the AFG system has workload advantages over previous systems such as MindTrail, for which the preparation time alone can exceed four hours (Stevens \& Jamieson, 2002), and does not increase workloads or require reductions in teaching loads. Such benefits would be 
expected to be even greater for information systems educators, as their increased ICT competence would reduce installation difficulties and improve ease of use.

The second important concern was that of feedback quality. This encompasses criteria such as timeliness (James et al., 2002; Wiggins, 1997), consistency (Holmes \& Smith, 2003), quantity (Holmes \& Smith, 2003), detail (Wiggins, 1997) and legibility (Higgins et al., 2002); it should also be informative (James et al., 2002), specific (Higgins et al., 2002) and personalised (Higgins et al., 2002). The results indicate that AFG improves quality across several of these criteria. Timeliness appeared to be the most important area of improvement noted by staff, being the most popular response to the qualitative question D1 and gaining the second highest mean of the UTAUT questions. Other improvements included greater consistency between markers, evidenced by several qualitative responses to D1; several further qualitative responses indicated that comment recycling was useful, suggesting that it may be possible for the quantity of feedback to be increased if educators spend less time repeating themselves. While one of the more experienced staff saw little benefit in standard comments, preferring instead to personalise all comments, another appreciated the benefits of being supplied with a range of starting comments by the course coordinator.

The results also show support for the use of the UTAUT model to assess the acceptance and use of technology. In particular, performance expectancy was rated very positively across the key areas of time required, costs and, to a lesser extent, quality of feedback. This shows that educators perceive that AFG would be more likely to be used to aid the user directly, through time and cost savings, than the recipients of the feedback. Facilitating conditions were rated highly, particularly specialised instructions, and effort expectancy was generally positive. Social influence appeared to have little impact in this situation, since the opportunity for it was very limited; the level of interaction between educators that was required by the course was restricted mainly to communication between the course coordinator and the educators who they had employed. Overall, performance expectancy was the highest rated construct, meaning that work improvements are seen as most positive. Satisfaction with the package as a whole also appeared to be high, with the intention for future use and recommendation to others being positively rated.

The results of this study also provide interesting insight into the generalisability, usability and potential adoption of AFG in different contexts. Results showed that on the key construct of effort expectancy, those educators with higher levels of expertise and experience with ICT expended less effort in installing and using the system. Hence adoption may be highest in faculties where staff have comparatively higher levels of ICT expertise.

Generalisability may also be considered in terms of the type of assessment and discipline to which AFG can be applied. For example, ICT can be classified as a "hard" applied discipline where answers to assessment tasks are often very objective, as compared to "soft" applied disciplines like management where assessments involve greater subjectivity (Dunn, Parry \& Morgan, 2002). In this study AFG was used to assess and provide feedback in a management course where the assessment type was essay, which generally involves more subjective feedback. Given the improvements in quality and savings in time and cost when used in a subjective discipline, these advantages may apply in even greater measure across more objective disciplines such as information systems, where standardisation of comments and marks tends to be more straightforward. 
The results of this exploratory study can also be triangulated with previous studies where student perceptions of feedback quality were measured. An investigation of an earlier version of AFG (Debuse et al., 2007) suggested that it can produce feedback of comparable quality from a student perspective, in terms of constructiveness, helpfulness and errors, to that produced using manual approaches or the more complex EFS package. The defunct MindTrail system has been found to produce feedback that is not significantly different to manual alternatives in terms of improvements gained in student marks through using the feedback provided, although it does appear to improve consistency between markers (Cargill, 2001).

Finally, the student cohort involved in this study included students studying in a variety of modes including on campus and online, part time and full time. The educators involved also represented a range of employment modes and experience. However, despite this breadth of coverage, caution should be exercised in generalising the results of this study given its exploratory nature.

This study suggests that automated feedback generation has the potential to provide compelling benefits, and given its limited sample size further research is required to determine whether the approach should be adopted within other universities. If these exploratory results are confirmed then educators are likely to benefit from increased marking speed, higher consistencies across markers and strong support for knowledge dissemination from faculty coordinating courses to the educators that they employ. Such research is further supported by past studies suggesting that students do not perceive any detriment to the quality of feedback if it is produced using automated approaches (Debuse et al., 2007); indeeed, standardising comments allows them to receive more detailed guidance, and the improvements in speed allow them to learn from their mistakes at an earlier stage in each course.

\section{Conclusions}

This exploratory research suggests that an automated feedback generator may be used to reduce workloads while simultaneously improving the quality of feedback provided to students in terms of timeliness, consistency and quantity. Combined with previous research indicating that systems such as AFG are capable of producing comparable results to manual approaches from a student perspective (Debuse et al., 2007), this study highlights the need for further investigation to confirm that automated feedback technology is capable of improving outcomes for both educators and students.

Automated feedback generation is ideally suited to an ICT faculty, since their technological expertise is likely to reduce the adoption barrier of installation and improve the ease of use. However, provided sufficient technical support is given there is no reason why such systems cannot be used in any faculty. Although such support may require significant resources, this can be offset against the efficiency benefits gained by automating feedback production, and it may be possible to argue that the net impact is positive rather than negative.

While this study has provided valuable insights into staff perceptions of an automated feedback system it has some limitations. Firstly the study was undertaken in a course with a total of eight educators, with considerable variation in responses for some items, and hence can only be considered as an exploratory investigation requiring further verification. Secondly, a specific comparison with a manual approach was not investigated. Finally, although the savings in time were measured, they were not 
quantified to determine the precise benefits accrued from automation. Future research should continue to monitor both student and educator perceptions of advantages and possible improvements to automated feedback systems, to provide further triangulation of these initial exploratory results.

\section{References}

Blayney, P. \& Freeman, M. (2004). Automated formative feedback and summative assessment using individualised spreadsheet assignments. Australasian Journal of Educational Technology, 20(2), 203-231. http:/ / www.ascilite.org.au/ajet/ajet20/blayney.html

Bongalos, Y., Bulaon, D., Celedonio, L., Guzman, A. d. \& Ogarte, C. (2006). University teachers' experiences in courseware development. British Journal of Educational Technology, 37(5), 695.

Bower, M. (2005). Online assessment feedback: Competitive, individualistic, or... preferred form! Journal of Computers in Mathematics and Science Teaching, 24(2), 121-147.

Cargill, M. (2001). Enhancing essay feed-back using 'MindTrail' software: Exactly what makes the difference in student development? Proceedings of the Changing Identities: Language and academic skills conference, the University of Wollongong, Australia. http: / / learning.uow.edu.au/LAS2001/ unrefereed/ cargill.pdf

Debuse, J., Lawley, M. \& Shibl, R. (2007). The implementation of an automated assessment feedback and quality assurance system for ICT courses. Journal of Information Systems Education, 18(4), 491-502.

Denton, P. (2001). MS Office software for returning feedback to students via email. [viewed 30 May 2005] http: / / www.heacademy.ac.uk/embedded_object.asp?id=21653\&filename=Denton

Dunn, L., Parry, S. \& Morgan, C. (2002). Seeking quality in criterion referenced assessment. Learning Communities and Assessment Cultures Conference. University of Northumbria.

Higgins, R., Hartley, P. \& Skelton, A. (2002). The conscientious consumer: Reconsidering the role of assessment feedback in student learning. Studies in Higher Education, 27(1), 53.

Holmes, L. E. \& Smith, L. J. (2003). Student evaluations of faculty grading methods. Journal of Education for Business, 78(6), 318.

James, R., McInnis, C. \& Devlin, M. (2002). Assessing learning in Australian universities: Ideas strategies and resources for quality in student assessment. Centre for the Study of Higher Education, University of Melbourne.

McCormack, C. \& Taylor, M. J. (2006). Electronic delivery of oral feedback on graphic design projects. Proceedings ASCILITE Sydney 2006. http: / / www.ascilite.org.au/conferences/sydney06/ proceeding/pdf_papers/p72.pdf

Meister, D. B. \& Compeau, D. R. (2002). Infusion of innovation adoption: An individual perspective. Proceedings of the ASAC, Winnipeg, Manitoba.

Parikh, A., McReelis, K. \& Hodges, B. (2001). Student feedback in problem based learning: A survey of 103 final year students across five Ontario medical schools. Medical Education, 35(7), 632-636.

Re:Mark (2006). Re:Mark online grading and markup solution for Blackboard. [viewed 22 May 2006] http: / / www.mydropbox.com/info/ReMark_for_Blackboard.pdf

Selwood, I. (2004). Information technology in educational administration management and in schools in England and Wales: Scope, progress and limits. Unpublished PhD, University of Birmingham.

Selwood, I. \& Pilkington, R. (2005). Teacher workload: using ICT to release time to teach. Educational Review, 57(2), 163-174.

Stevens, K. \& Jamieson, R. (2002). The introduction and assessment of three teaching tools (WebCT, MindTrail, EVE) into a post graduate course. Journal of Information Technology Education, 1(4), 233-252.

Venkatesh, V. \& Davis, F. (2000). A theoretical extension of the technology acceptance model: Four longitudinal field studies. Management Science, 45(2), 186-204. 
Venkatesh, V., Morris, M., Davis, G., \& Davis, F. (2003). User acceptance of information technology: Toward a unified view. MIS Quarterly, 27(3), 425-478.

Weaver, M. R. (2006). Do students value feedback? Student perceptions of tutors' written responses. Assessment and Evaluation in Higher Education, 31(3), 379-394.

Wiggins, G. (1997). Feedback: How learning occurs. In Assessing impact evidence and action (pp. 31-39): American Association of Higher Education.

Williams, R., \& Dreher, H. (2004). Automatically grading essays with MarkIT. Journal of Issues in Informing Science and Information Technology, 1, 693-700.

\section{Appendix 1: Sample feedback document}

Research Report

Smith John 123456

\begin{tabular}{|c|c|c|}
\hline Criteria & Marks & Comments \\
\hline \multicolumn{3}{|r|}{ Presentation } \\
\hline Communication & $5 / 5$ & $\begin{array}{l}\text { You need to use the Harvard style } \\
\text { Try to avoid direct quotations as much as possible } \\
\text { Good, up to date references } \\
\text { Excellent range of references } \\
\text { Publications that app ear within ProQuest should only } \\
\text { have details relating to their publication outlet reported } \\
\text { so for example the ProQuest number and URL should be } \\
\text { omitted } \\
\text { No problems here }\end{array}$ \\
\hline \multicolumn{3}{|r|}{ Problem Definition } \\
\hline Literature Review & $6 / 10$ & $\begin{array}{l}\text { The articles you have used are too outdated } \\
\text { Try to tie the papers into your own arguments, rather than } \\
\text { presenting so much detail on each example } \\
\text { Greater synthesis of concepts is required } \\
\text { You have devoted too much space to this section } \\
\text { The focus needs to be kept on the research topic here }\end{array}$ \\
\hline Research Questions & $0 / 20$ & No research questions have been presented \\
\hline \multicolumn{3}{|r|}{ Other Criteria } \\
\hline Analysis & $30 / 30$ & \\
\hline \multicolumn{3}{|r|}{ Total } \\
\hline Total Marks & $44 / 70$ & \\
\hline
\end{tabular}

\section{Appendix 2: Survey}

See http: / / www.ascilite.org.au/ ajet/ ajet24/ debuse-appendix2.pdf (Copyright: The authors)

Dr Justin Debuse, Lecturer, Dr Meredith Lawley, Associate Professor and Rania Shibl, Associate Lecturer, Faculty of Business, University of the Sunshine Coast,

Maroochydore DC, Australia. Web: http:/ / www.usc.edu.au/

Email: jdebuse@usc.edu.au, mlawley1@usc.edu.au, rshibl@usc.edu.au 